\title{
Correlation between the Differences between the Two Adjacent Pulse Wave Velocities and Body Mass Index
}

\section{Guanghui Qin}

The Third Affiliated Hospital of Naval Military Medical University (Shanghai Oriental Hepatobiliary Surgery Hospital), Shanghai, China

Email: banmao007@163.com

How to cite this paper: Qin, G.H. (2022) Correlation between the Differences between the Two Adjacent Pulse Wave Velocities and Body Mass Index. Open Access Library Journal, 9: e8290.

https://doi.org/10.4236/oalib.1108290

Received: December 11, 2021

Accepted: January 10, 2022

Published: January 13, 2022

Copyright $\odot 2022$ by author(s) and Open Access Library Inc.

This work is licensed under the Creative Commons Attribution International License (CC BY 4.0).

http://creativecommons.org/licenses/by/4.0/

(c) (i) Open Access

\begin{abstract}
Objective: To investigate the correlation between the differences between the two adjacent pulse wave velocities and body mass index (BMI). Methods: Body mass index and pulse velocity difference were measured in 60 patients. Results: The difference of pulse wave velocity between adjacent two times in patients with $\mathrm{BMI}<24$ was less than that in patients with BMI $\geq 24$, the difference was statistically significant. Conclusion: Pulse velocity difference is related to body mass index.
\end{abstract}

\section{Subject Areas}

Diagnostics

\section{Keywords}

Pulse Velocity Difference, Difference between Two Adjacent Pulse Wave Velocities, daPWV, Body Mass Index, BMI

\section{1. 引言}

个体内部脉搏波传导速度(PWV)之间的差异可能具有临床意义 [1], 针对 这一假说，有一项研究开展了间接的带有主观性的初步验证，表明脉速差(相 邻两次脉搏波传导速度之间的差异, The differences between the two adjacent pulse wave velocities, 简称脉速差, daPWV)与痛经疼痛程度正相关, 该差异 具有临床意义[2]。脉速差这个指标由本文作者发现并首次提出。在仪器生产 方香港生物医药控股有限公司的支持下，世界上首台可以测量脉速差的脉搏 波传导速度检测仪迅速应用到了临床。基于这台仪器，本文开展了脉速差与 
体重指数(BMI)的相关性研究。

\section{2. 临床研究资料}

\section{1. 一般资料}

2016.2 2018.4 月复旦大学附属金山医院门诊患者 60 例, 年龄 23 82 岁, 疾病背景未调查。非超重组 31 例, 男性 5 例, 女性 26 例; 超重组 29 例, 男 性 9 例, 女性 20 例。非超重组年龄 $59 \pm 12.65$ 岁, 超重组年龄 $57.62 \pm 14.04$ 岁。两组性别、年龄经统计学检验无差异。

\section{2. 体重指数标准}

依据中国肥胖问题工作组数据汇总分析协作组的研究成果 [3], 以 BMI $<$ 24 为非超重, $B M I \geq 24$ 为超重。本研究以此将患者分为超重组和非超重组。

\section{3. 方法}

\section{1. 脉速差的数值的获得方法}

现有的脉搏波传导速度测定仪一般是对一个节段的动脉连续测量一定时 间获得若干个脉搏波传导速度, 取其平均值, 在结果输出方面只显示了平均 值, 用这个平均值来评价动脉硬化等疾病, 而每个脉搏波传导速度的原始数 值则隐藏在软件的数据库中。只要将软件进行相应的修改, 基于仪器获得每 个脉搏波传导速度的原始值, 比较相邻两次脉搏波传导速度之间的差异(脉速 差)，取这些差异的平均值和最大值，来进行临床研究。

\section{2. 检测方法}

采用脉搏波检测仪(AS-2000，香港医药生物控股有限公司生产)，患者安 静休息 5 分钟后平躺在检查床上, 将传感器固定在颈动脉、双手桡动脉、双 足背动脉、右腿股动脉搏动处, 仪器自动进行检测。同时将患者身高、体重 输入仪器, 自动获得 BMI 数值。

\section{3. 统计学方法}

应用 SPSS17.0 统计软件进行分析。计量资料以均数 \pm 标准差描述, 用 $\mathrm{t}$ 检验。计数资料采用卡方检验。

\section{4. 结果}

两组患者主动脉脉速差的最大值、平均值比较, 超重组大于非超重组, 差异有统计学意义。见表 1 。

两组患者左上肢脉速差的最大值、平均值比较, 超重组大于非超重组, 差异有统计学意义。见表 2 。

两组患者右上肢脉速差的最大值、平均值比较, 超重组大于非超重组, 差异有统计学意义。见表 3 。

两组患者左下肢脉速差的最大值、平均值比较, 超重组和非超重组无差 
异。见表 4。

两组患者右下肢脉速差的最大值、平均值比较, 超重组和非超重组无差 异。见表 5 。

表 1. 两组患者主动脉脉速差的最大值、平均值比较 $(\bar{X} \pm s)$ (单位: $\mathrm{m} / \mathrm{s}$ )

\begin{tabular}{cccccc}
\hline 组别 & $\mathrm{n}$ & 最大值 & 平均值 & $\mathrm{T}$ & $\mathrm{P}$ \\
\hline 非超重组 & 31 & $0.78 \pm 0.68^{*}$ & $0.30 \pm 0.23^{*}$ & -3.28 & 0.002 \\
超重组 & 29 & $1.64 \pm 1.27$ & $0.67 \pm 0.53$ & -3.48 & 0.001 \\
\hline
\end{tabular}

注: 两组相比, $\mathrm{P}<0.05$, 差异具有统计学意义。

表 2. 两组患者左上肢脉速差的最大值、平均值比较 $(\bar{X} \pm s)$ (单位: $\mathrm{m} / \mathrm{s}$ )

\begin{tabular}{cccccc}
\hline 组别 & $\mathbf{n}$ & 最大值 & 平均值 & $\mathrm{T}$ & $\mathrm{P}$ \\
\hline 非超重组 & 31 & $1.51 \pm 1.49^{*}$ & $0.57 \pm 0.50^{*}$ & -2.43 & 0.018 \\
超重组 & 29 & $2.68 \pm 2.20$ & $1.08 \pm 0.91$ & -2.71 & 0.009 \\
\hline
\end{tabular}

注: 两组相比, $\mathrm{P}<0.05$, 差异具有统计学意义。

表 3. 两组患者右上肢脉速差的最大值、平均值比较 $(\bar{X} \pm s)$ (单位: $\mathrm{m} / \mathrm{s}$ )

\begin{tabular}{cccccc}
\hline 组别 & $\mathbf{n}$ & 最大值 & 平均值 & $\mathrm{T}$ & $\mathrm{P}$ \\
\hline 非超重组 & 31 & $1.43 \pm 1.57^{*}$ & $0.53 \pm 0.52^{\star}$ & -2.14 & 0.019 \\
超重组 & 29 & $2.60 \pm 2.14$ & $1.03 \pm 0.84$ & -2.80 & 0.007 \\
\hline
\end{tabular}

注: 两组相比, $\mathrm{P}<0.05$, 差异具有统计学意义。

表 4. 两组患者左下肢脉速差的最大值、平均值比较 $(\bar{X} \pm s)$ (单位: $\mathrm{m} / \mathrm{s}$ )

\begin{tabular}{cccccc}
\hline 组别 & $\mathbf{n}$ & 最大值 & 平均值 & $\mathbf{T}$ & $\mathbf{P}$ \\
\hline 非超重组 & 31 & $0.74 \pm 0.59^{*}$ & $0.32 \pm 0.30^{*}$ & -0.25 & 0.80 \\
超重组 & 29 & $0.77 \pm 0.47$ & $0.34 \pm 0.25$ & -0.24 & 0.80 \\
\hline
\end{tabular}

注: 两组相比, $\mathrm{P}>0.05$, 差异无统计学意义。

表 5. 两组患者右下肢脉速差的最大值、平均值比较 $(\bar{X} \pm s)$ (单位: $\mathrm{m} / \mathrm{s}$ )

\begin{tabular}{cccccc}
\hline 组别 & $\mathbf{n}$ & 最大值 & 平均值 & $\mathbf{T}$ & $\mathbf{P}$ \\
\hline 非超重组 & 31 & $0.75 \pm 0.58^{*}$ & $0.31 \pm 0.25^{*}$ & -0.32 & 0.75 \\
超重组 & 29 & $0.79 \pm 0.37$ & $0.34 \pm 0.14$ & -0.48 & 0.64
\end{tabular}

注: 两组相比, $\mathrm{P}>0.05$, 差异无统计学意义。

\section{5. 结论}

本次研究的结果表明: 主动脉、左上肢动脉、右上肢动脉三条动脉脉速 差的最大值、平均值均与体重指数相关, 以 24 为界, BMI 指数小于 24 的非 超重组, 脉速差小于超重组, 超重和肥胖是导致相邻两次脉搏波传导速度产 生差异的一个因素。由于超重和肥胖对健康是一种危害因素, 因此脉速差这 
个指标是人体的一种病态信号, 需要加以识别和调控。本次研究样本量小, 疾病背景也未考虑, 结论可能具有局限性。希望国内医学研究人员和仪器生 产厂家进一步努力, 尽快将脉速差这个指标进行规范化研究, 向世界推广这

一成果, 更好地为临床和患者服务。

本次研究同样也为一种涩脉客观诊断标准提供了客观支持。这种涩脉客 观诊断标准 [4] [5] [6]是：在心律律齐的情况下, 前后(相邻)两次脉搏波传导 速度有差异(即脉速差大于零)的脉是涩脉, 前后(相邻)两次脉的搏动位置有差 异的脉是澀脉, 差异越大, 代表涩的程度越大。本次研究发现, 超重、肥胖 的人群脉速差比非超重的人要大, 表明超重、肥胖的人群容易产生涩脉, 并 且澀的程度比非超重者大。这和中医传统理论所论述的涩脉认识相符合。传 统理论认为, 肥人多痰, 痰凝血瘀, 容易导致涩脉。因此, 本次研究表明, 文献 [4] [5] [6]所提到的涩脉客观诊断标准与传统理论的涩脉相符。本次研究 发现上肢的脉速差与体重指数相关, 下肢的脉速差与体重指数无相关, 与古 人主要在上肢桡动脉搏动部位寸口进行脉诊的诊疗习惯具有一致性。

目前只是孤立地研究某一节段动脉的脉搏波传导速度的平均值, 没有去 关注这一节段动脉各个脉搏波传导速度之间的差异。在一些患者身上，可以 检测到某一节段动脉的脉搏波传导速度存在快慢不一的现象, 所以需要连续 检测多个脉搏波传导速度, 取平均值来评估动脉硬化程度。而脉搏波传导速 度这种快慢不一的现象, 是身体的健康状态和病理状态的反应, 因此有必要 研究脉搏波传导速度这种快慢不一的现象。脉速差是指相邻两次脉搏波传导 速度之间的差异, 是研究这种快慢不一现象的一个指标。

脉速差作为一个客观可以测量的指标, 在现代医学领域有可能成为评价 疼痛程度、心血管危险程度等的指标。同时在中医领域, 脉速差是反应脉搏 流利度的一个客观指标, 脉搏的流利度是涩脉的特征[7], 因此其有可能是中 医澀脉的客观指标。

\section{基金项目}

本文获上海市科委课题(20Y21900800)资助。

\section{Conflicts of Interest}

The author declares no conflicts of interest.

\section{References}

[1] 覃光辉. 个体内部脉搏波传导速度之间的差异的初步认识[J]. 中国老年保健医学 杂志, 2014(1): 90.

[2] 覃光辉. 相邻两次脉搏波传导速度差异与疼痛程度的相关性研究 [J]. 中国老年保 健医学, 2014(3): 58.

[3] 中国肥胖问题工作组数据汇总分析协作组. 我国成人体重指数和腰围对相关疾 病危险因素异常的预测价值: 适宜体重指数和腰围切点的研究 [J]. 中华流行病学 杂志, 2002(1): 10-15.

[4] 覃光辉. 从物体运动状态认识涩脉的本质 [J]. 中医药导报, 2014(15): 95.

[5] 覃光辉, 薛轶燕, 王骁, 苏励, 李振龙, 蒋美艳. 一种涩脉客观诊断标准的初步验 
证研究[J]. 中医临床研究, 2014, 6(29): 52-53.

[6] 覃光辉. 涩脉新解[J]. 中医药导报, 2014, 20(1): 99.

[7] 张佳琪, 刘佳, 周灵运, 汪南玥. 涩脉形态特点剖析[J]. 中华中医药杂志, 2017, 32(6): 2394-2396.

\section{Appendix (Abstract and Keywords in Chinese) 脉速差与体重指数的相关性研究}

摘要: 目的: 调查脉速差与体重指数 $(\mathrm{BMI})$ 的相关性。方法: 对 60 例患者进 行体重指数和脉速差的检测。结果: $\mathrm{BMI}<24$ 的患者相邻两次脉搏波传导速 度的差异值小于 $\mathrm{BMI} \geq 24$ 的患者, 差异具有统计学意义。结论: 脉速差与体 重指数相关。

关键词: 脉速差, 相邻两次脉搏波传导速度之间的差异, daPWV, 体重指数, BMI 